\title{
Bioinspired neuron-like electronics
}

\author{
Xiao Yang ${ }^{1, \dagger}$, Tao Zhou ${ }^{1, \dagger}$, Theodore J. Zwang ${ }^{1, \dagger}$, Guosong Hong ${ }^{1}$, Yunlong Zhao ${ }^{1}$, Robert \\ D. Viveros ${ }^{2}$, Tian-Ming Fu' ${ }^{1}$, Teng Gao ${ }^{1}$, and Charles M. Lieber ${ }^{1,2,3,{ }^{*}}$ \\ ${ }^{1}$ Department of Chemistry and Chemical Biology, Harvard University, Cambridge, Massachusetts, \\ USA. \\ 2John A. Paulson School of Engineering and Applied Sciences, Harvard University, Cambridge, \\ Massachusetts, USA. \\ ${ }^{3}$ Center for Brain Science, Harvard University, Cambridge, Massachusetts, USA.
}

\section{Abstract}

\begin{abstract}
As an important application of functional biomaterials, neural probes have contributed substantially to studying the brain. Bioinspired and biomimetic strategies have begun to be applied to the development of neural probes, although these and previous generations of probes have had structural and mechanical dissimilarities from their neuron targets that lead to neuronal loss, neuroinflammatory responses and measurement instabilities. Here we present a bioinspired design for neural probes, neuron-like electronics (NeuE), where the key building blocks mimic the subcellular structural features and mechanical properties of neurons. Full three-dimensional mapping of implanted NeuE/brain interfaces highlights the structural indistinguishability and intimate interpenetration of NeuE and neurons. Time-dependent histology and electrophysiology studies further reveal a structurally and functionally stable interface with the neuronal and glial networks shortly following implantation, thus opening opportunities for next-generation brainmachine interfaces. Finally, the NeuE subcellular structural features are shown to facilitate migration of endogenous neural progenitor cells, thus holding promise as an electrically active platform for transplantation-free regenerative medicine.
\end{abstract}

The design of materials using concepts inspired by and/or mimicking biology is an attractive strategy for the development of innovative materials, and has led to exciting advances across a variety of fields, including controlling crystallization kinetics ${ }^{1}$, developing mechanically

\footnotetext{
*Correspondence to: $\mathrm{cml} @$ cmliris.harvard.edu.

$\dagger$ These authors contributed equally to this work.

Author contributions

X.Y. and C.M.L. designed the experiments. X.Y., T.Z., T.J.Z., G.H., Y.Z., R.D.V., T.-M.F. and T.G. performed the experiments. X.Y., T.Z., T.J.Z. and C.M.L. analyzed the data. X.Y. and C.M.L. wrote the paper. All authors discussed the results, revised or commented on the manuscript.

Reporting Summary. Further information on research design is available in the Nature Research Reporting Summary linked to this article.

Code availability. The code used for data analysis is available from the corresponding author upon reasonable request.

Data availability

The data that support the findings of this study are available from the corresponding author upon reasonable request.

Competing interests

The authors declare no competing interests.
} 
tough materials ${ }^{2}$, and engineering the design and functionality of biomaterials ${ }^{3,4}$.

Biomaterials have also been used in the development of multifunctional neural probes that will help to understand the brain ${ }^{5,6}$. Recently, the design of implanted neural probes has begun to benefit from the advantages and opportunities afforded by bioinspired and biomimetic design strategies ${ }^{7}$. These strategies include designing a stimuli-responsive material with dynamic stiffness ${ }^{8}$ for easy insertion of probes, and utilizing vein compression to reduce tissue trauma ${ }^{9}$. In view of these successes in implementing bioinspired concepts to neural interfacing, it is noteworthy that neural probes that adapt the structural or mechanical features of cells have not been explored.

Evidence suggests that structural and mechanical differences between neural probes and neuron targets in the brain can lead to disruption of the native tissue ${ }^{10,11}$ that negatively impacts the capability to stably interrogate and modulate natural physiological activity over time ${ }^{5,6,12-15}$. Research aimed at reducing these disparities has focused on improving mechanical properties by optimizing device geometry ${ }^{12,16-20}$ or using more flexible materials ${ }^{21}$, including mesh probes that achieve tissue-like flexibility ${ }^{16-18}$. Nevertheless, existing probes remain essentially 'foreign' to the primary neuron building blocks of the brain, in terms of structure and mechanics, posing a dichotomy with the successful concepts of bioinspired design.

\section{Design and characteristics of neuron-like electronics}

As a step towards biomimetic electronics that address the baseline distinctions between implanted probes and the fundamental neuron component of the brain, we have focused on a probe unit building block that is structurally and mechanically similar to a neuron at the subcellular level (Fig. 1a), although not yet with the functional complexity. In our biomimetic design the sizes of the metal recording electrode and interconnect, which constitute the probe building block, match those of the soma and neurite of a typical pyramidal neuron. Moreover, the interconnect and axon have similar flexibility (details are provided in the following), and the thin polymer insulation is analogous to the myelin sheath; both assist in the propagation of electrical signals from the electrode and soma, respectively. Finally, the neuron-like building blocks are organized to construct an open three-dimensional (3D) electronic network, neuron-like electronics (NeuE), with structure and topology similar to neural networks (Fig. 1a, inset).

The NeuE was fabricated using photolithography ${ }^{17}$ where the neurite-like interconnects consist of polymer/metal/polymer structures with polymer and metal widths of 4 and 2 or 1 and $0.6 \mu \mathrm{m}$, respectively, with a total thickness of ca. $0.9 \mu \mathrm{m}$ for the two designs studied (Supplementary Table 1 and Methods). The soma-like platinum recording electrodes were supported on the lower polymer layer (Supplementary Fig. 1). Images of a pyramidal neuron and the two different building blocks used for the NeuE (Fig. 1b, Supplementary Fig. 2a,b and Methods) highlight the biomimetic structural characteristics, including the similarity in sizes of the smaller unit interconnect with dendritic branches. The fabrication of NeuE is detailed in Methods and key probe layers are shown in Supplementary Fig. 1. 
A key physical consequence of the neuron-like dimensions of the NeuE building blocks is that the mechanical stiffness is reduced 5-20 times compared to the most flexible state-ofthe-art reported probes ${ }^{17,19,20,22}$ due to the reduction in feature widths to match the diameter of neurites (Fig. 1c and Supplementary Note 1). The calculated bending stiffness of the NeuE neurite-like interconnect is comparable to the axon of a neuron (Fig. 1c and Supplementary Note 1$)^{23,24}$, thus matching the flexibility of a subcellular component for the first time. Also, the estimated percentage of occupied volume for the implanted NeuE probes, $0.07-0.3 \%$ (Supplementary Table 1 and Supplementary Note 2) for the designs in Fig. 1b, is distinct from conventional solid probes ${ }^{5,6}$ that exclude all cells from their occupied volume, and is up to 20 times lower than recent macroporous mesh probes ${ }^{17}$. Here we focus on 16-channel NeuE probes, but note that higher channel numbers should be achievable without sacrificing the flexibility and porosity by using higher-resolution fabrication to integrate multiple metal interconnect lines within a given neurite-like element $^{25,26}$.

\section{Similar structure and 3D interpenetration with neurons}

We asked how these unique features of NeuE probes affect the interface with neurons within the brain by full 3D mapping of the probe and surrounding brain tissue with subcellular resolution (Supplementary Fig. 3 and Methods) at different times post-implantation. We covalently bound a rhodamine derivative within the bulk of the polymer layers of the NeuE probes to provide stable conjugation and fluorescence under physiological conditions (Supplementary Fig. 4 and Methods). Precise targeted delivery of NeuE probes into live mice was performed using a stereotaxic injection method described previously (see Methods) ${ }^{27}$. The NeuE probes were implanted into the hippocampus (HIP) through cortex (CTX) of transgenic mouse lines expressing either yellow fluorescent proteins (YFP) in neurons (Thy1-YFP-H) ${ }^{28}$ or green fluorescent proteins (GFP) in astrocytes (GFAP-GFP) ${ }^{29}$. A representative 3D reconstructed image of the NeuE and neurons at 6 weeks post-injection (Fig. 1d and Supplementary Video 1) highlights the similar sizes of the neurite-like interconnects and neuron neurites, flexibility of these biomimetic 'neurites' and interpenetration of the NeuE and neurons from CTX through CA3 region of the HIP. These and other key features are even more evident in higher-resolution images of the CTX (Fig. 1e, I), CA1 (Fig. 1e, II) and CA3 (Fig. 1e, III), which show the structural similarity between recording electrodes and neuron soma, as well as the interpenetrating interconnects and neurites. The capability to spatially resolve electrodes (white dashed circles in Fig. 1e) relative to surrounding neurons in situ with micron resolution allows for correlating structural and functional mapping, as will be discussed later, and has not been possible previously for implanted multi-electrode neural probes.

The structural similarity and interpenetration of the NeuE probe with neurons were further examined by color-encoding their positions as a function of depth within 3D images. Comparison of zoomed-in views of CA1 with standard fluorophore (Fig. 1f,I) and depthcoding (Fig. 1f,II and Supplementary Video 2) indicates that NeuE integrates in neural tissue without noticeable disturbance, and indeed, it is difficult to distinguish the interwoven NeuE and neuron structures. Examination of neurite-like interconnects and neuron neurites (Fig. 1f,III,IV and Supplementary Video 3) highlights this latter point, which emphasizes the 
structural biomimetic nature of the NeuE, and strikingly, shows that neuron neurites and NeuE neurite-like interconnects make close contact that might in future be developed into artificial neuro-electronics junctions or synapses. In addition, 3D images (Fig. 1g) of the smaller NeuE design (Fig. 1b,III) at 2 weeks post-implantation show similar integration, that the smaller neurite-like interconnects are comparable to dendritic branches (white asterisks), and show that the overall NeuE probes exhibit greater flexibility within the tissue, consistent with its smaller bending stiffness.

\section{Time-dependent brain interface}

We also asked how the implanted NeuE affects the distribution of key cell types across the HIP and CTX as a function of time post-implantation in Thy1-YFP-H and GFAP-GFP transgenic mice, which have been previously validated for assessing tissue integration and foreign body response on the basis of in vivo and in vitro imaging studies ${ }^{22,28,29}$. Histology imaging and quantitative analyses have been replicated on multiple $(\mathrm{N}=3)$ independent samples containing NeuE probes at 2 days, 2 weeks, 6 weeks and 3 months postimplantation for both transgenic mouse lines, and show similar results, as detailed in the following (Fig. 2 and Supplementary Figs. 5-7). Representative 3D images of NeuE/neuron (Fig. 2a and Supplementary Fig. 5a) and NeuE/astrocyte (Fig. 2b and Supplementary Fig. $5 b)$ interfaces show a relatively uniform cell distribution without obvious depletion of neurons or enhancements of astrocytes near the NeuE from the earliest time of analysis onward.

We quantified the time-dependent post-implantation response by analyzing the cell distributions in volumes containing NeuE and the dentate gyrus (DG), CA1, or the CTX (Fig. 2c and Supplementary Note 4). Representative tissue volumes (Supplementary Fig. 8a) encompass the NeuE probe and most of the cells in each of these three brain regions.

Although some displacement of existing tissue occurs during insertion of the glass capillary, which is withdrawn from the brain tissue during the injection of the NeuE, normalized neuron fluorescence intensity (Fig. 2c) demonstrated there is substantial neuronal density in the interior of the NeuE probe as early as 2 days. Moreover, the neurons exhibit a fully endogenous distribution in the DG, CA1 and CTX regions at times extending from 2 weeks. Analyses of astrocyte labelled samples (Fig. 2d) further show the astrocyte density is close to baseline at 2-14 days, and is uniform at endogenous levels at longer times. These results for neurons and astrocytes are quantitatively similar to control samples of the same brain regions in the absence of NeuE implantation (Supplementary Fig. 9 and Supplementary Note 4). We repeated the time-dependent histology studies for two distinct sets of Thy 1-YFP-H and GFAP-GFP mice at 2 days, 2, 6 and 12 weeks (16 mice in total; Supplementary Table 2). Representative fluorescence images (Supplementary Fig. 6) and quantitative analyses (Supplementary Fig. 7) demonstrate reproducibility of these results. Comparison of the normalized fluorescence intensity from tissue volumes (Supplementary Fig. 8a) versus thin horizontal slices (Supplementary Fig. 8b) relative to the NeuE probes for both neurons and astrocytes also show similar overall results, although there are substantially smaller fluctuations in the fluorescence intensities analyzed on the 3D volume samples (Supplementary Fig. 8c,d). 
In addition, the samples shown in Fig. $2 \mathrm{~b}$ and Supplementary Fig. $5 \mathrm{~b}$ were subject to tissue clearing ${ }^{30,31}$ followed by microglia immunostaining (see Methods). Fluorescence images (Supplementary Fig. 5c) and quantitative fluorescence intensity analyses (Supplementary Fig. 5d) show a uniform distribution of microglia around NeuE probes through the CTX, CA1 and DG over this time period, consistent with the absence of an immune response inferred from analyses of neuron and astrocyte distributions. Additionally, sham injection control experiments and subsequent immunostaining (Supplementary Fig. 10 and Methods) show a minimal immune response, as revealed by the close to endogenous levels of astrocytes and microglia at the site of injection.

These data contrast the typical depletion of neurons and proliferation of astrocytes and microglia close to the external boundary of solid probes ${ }^{10,11}$, as well as the decrease in neuron density within the interior and increase in astrocyte density at the surface boundary of conventional macroporous mesh probes even after 2 weeks post-implantation ${ }^{18}$. Hence, the full 3D analyses provide a quantifiable measure of the substantially lower invasiveness of the NeuE probes. We suggest that the bioinspired design, which mimics the size features and mechanical properties of neurons as well as occupying less than $0.3 \%$ volume once implanted (Supplementary Table 1), is crucial to achieving the observed results. Specifically, these features may allow for the migration of cells and the diffusion and dispersion of proinflammatory signaling proteins ${ }^{32}$ that ultimately leads to this attractive behavior of not activating astrocytes or microglia.

\section{Stable single-unit recording following implantation}

We asked whether it would be possible to follow the activity of the same neurons from the time of implantation onward and, moreover, to correlate the measured activity with the subcellular resolution topology of the imaged NeuE and neurons in 3D. NeuE probes were implanted with 16 addressable electrodes distributed across the HIP and CTX, and the probe/brain was imaged at the 3-month recording endpoint to spatially resolve the barcoded electrodes with respect to the surrounding neurons (Supplementary Fig. 11 and Methods). Multiplexed recordings (Fig. 3a and Supplementary Fig. 12) exhibit stable single-unit spikes across the HIP through the CTX regions from the time of the initial implantation (day 0) through to the endpoint of the measurements (day 90), where the position of the NeuE probe was fixed following initial implantation. This contrasts with the position adjustments often used to reacquire active neuron signals between recording sessions for conventional probes $^{33,34}$. Overall analyses of the single-unit spike signal-to-noise ratios (SNRs; Supplementary Fig. 13) show nearly constant values from the time of implantation onward. In addition, the electrode interfacial impedances exhibited relatively constant values over time, and the inter-channel impedances show minimal crosstalk between channels (Supplementary Fig. 14 and Methods).

Given this global single-unit recording stability, we asked whether it was possible to illuminate the single neuron activity as a function of time by tracking isolated neurons (Supplementary Note 5). Comparison of sorted spikes from each channel for days 0, 7, 14 and 90 (Fig. 3b and Supplementary Fig. 15) highlight several points. First, the isolated neurons, which are highlighted by different color spike waveforms in each of the 16 
channels, are remarkably constant over the three months of recording. There is no evidence for loss of isolated neuron signals over this period, although new neuron signals are observed in 11 out of 16 channels during the first 2 weeks (Fig. 3b,c and Supplementary Fig. 15). These results contrast the decrease in recorded neurons with time for conventional neural probes ${ }^{34,35}$, as well as the ca. 1-month period necessary to first see stabilized and well-isolated single units with mesh electronics ${ }^{17}$. Second, the number of recorded neurons varies across channels in a way that correlates with the neuron soma density in different HIP subfields.

Third, we have carried out analyses to assess the strength of our above conclusion based on sorted neuron spikes. Principal component analysis (PCA) ${ }^{35,36}$ shows the same clusters with relatively constant positions in the first and second principal component plane (PC1-PC2) over the 12 weeks studied (Supplementary Fig. 16), thus demonstrating single-unit stability over time. In addition, L-ratio analysis (Supplementary Note 5) ${ }^{37,38}$ shows values $<0.05$ for all detected neurons (Supplementary Table 3) and indicates minimal contamination between isolated units and thus good unit separation. Furthermore, quantitative waveform autocorrelation analysis for the same neuron recorded on different days (Supplementary Fig. 17 and Supplementary Note 5) ${ }^{34}$ demonstrates that the neuron spikes have consistent characteristic waveform features over time which is indicative of chronic recording stability.

Finally, we repeated the time-dependent electrophysiology studies and analyses for two additional mice implanted with NeuE probes (Supplementary Figs. 18 and 19). These results are consistent with the data presented in Fig. 3 (and Supplementary Figs. 12-17) with a total of 40 initially connected channels demonstrate the reproducibility of the stable single neuron recording and tracking of NeuE probes. Furthermore, analyses of mechanical stress in deformed NeuE elements characterized in 3D images of the implanted probes demonstrated that the maximum stress is well below the fatigue failure limit of SU- 8 material ${ }^{39}$ used in fabrication (Supplementary Note 6), thus indicating that NeuE probes could exhibit longterm mechanical robustness beyond the 3 months studied.

The combined electrophysiology and imaging data allowed us to ask whether the intact interface between the NeuE electrodes and neurons across the HIP and CTX could allow the spatial coordinates of the same spiking neuron detected by three nearby recording electrodes to be determined by triangulation (Supplementary Note 5$)^{40,41}$. Triangulation has been used previously ${ }^{40,41}$, although it has not been possible to register the electrode/neuron positions at the cellular scale as probes must be extracted for histology studies $33,34,40$. An example is that of electrodes 12, 13 and 14, which record the same neuron (Fig. 3d); this was identified (Fig. 3d, II) from the population of CA1 neurons surrounding these electrodes (Fig. 3d, I) by in situ reconstruction of the 3D electrodes/neurons interface. We suggest this capability of mapping recorded cells onto the undisturbed imaged brain structures could open new opportunities to link directly functional and topological circuits within their physiological context. This is distinct from reported methods combining in vivo imaging and subsequent in vitro brain slice electrophysiology where additional neuron transduction for structural marker expression ${ }^{42}$ or fluorescent microspheres ${ }^{43}$ are required to re-identify the same neurons. Moreover, this registration-free in situ 3D mapping capability opens the possibility of directly correlating experience-dependent neuronal activity with the underlying neuronal 
connectivity when combined with recent neuronal activity markers such as immediate early genes $^{44}$.

\section{NeuE promotes the migration of newborn neurons}

The 3D interpenetration of neurons through NeuE probes and the observation of new spiking neuron signals at early times led us to ask whether native neural progenitor cells (NPCs) originating from the subgranular zone (SGZ) of the HIP 45,46 migrate and concurrently develop into newborn neurons along the neurite-like structure of the NeuE since probes are implanted with ends touching or spanning the SGZ (Fig. 4a inset and Supplementary Fig. 20a). The presence of NPC-derived newborn neurons was assessed by staining NeuEimplanted tissue samples with doublecortin (DCX) antibody for newborn neurons ${ }^{45,46}$ and 4',6-diamidino-2-phenylindole (DAPI) for nuclear DNA, following tissue clearing (see Methods) ${ }^{30,31}$. Representative 3D images of brain tissue at 1 week post-implantation (Fig. 4a,b) show substantial $\mathrm{DCX}^{+}$cells associated and aligned with the NeuE structure, yet little or no DCX staining of $\mathrm{DAPI}^{+}$cells farther away from the probe.

To gain further insight into the importance of the NeuE biomimetic structure in promoting the association and migration of $\mathrm{DCX}^{+}$newborn neurons, we also assessed NeuE-injected samples collected at 2 weeks post-implantation (Supplementary Fig. 20) and controls using the significantly larger (20 $\mu \mathrm{m}$ width features) mesh electronics (Supplementary Fig. 21). Overall, there is a statistically significant decrease $(P<0.01)$ in the enhancement of $\mathrm{DCX}^{+}$ cells near NeuE at 2 weeks versus 1 week (Fig. 4c, Supplementary Fig. 20 and Supplementary Note 4$)$ and, strikingly, the $20 \mu \mathrm{m}$ control mesh shows a substantial approximately threefold decrease $(P<0.001)$ in $\mathrm{DCX}^{+}$cells compared to NeuE at both time points (Fig. 4c, Supplementary Fig. 21 and Supplementary Note 4). These quantitative data suggest that the biomimetic neurite-like topographical features of NeuE may modulate the endogenous NPC-derived cell behavior including adhesion and migration ${ }^{47}$, and help to explain the rapid tissue healing and the emergence of 'new' spiking neurons.

\section{Summary and outlook}

The NeuE represents a strong case for the use of bioinspiration and biomimicry as tools for the design of innovative materials and devices for neurotechnology. In contrast to other state-ofthe-art electrophysiology probes ${ }^{12-15,17-20,22,34,35}$, NeuE probes match the subcellular feature sizes and mechanical properties of the neurons. NeuE probes elicit negligible immune response compared to other probes ${ }^{12,14,17-20}$, and exhibits seamless interpenetrating interfaces with the brain from day-2 post-implantation onward. In addition, NeuE probes demonstrate stable single-unit recording of individual cells in the nearly native physiological context without loss in recording quality from shortly after implantation to at least 3 months. We suggest these characteristics arise from matching structural and mechanical properties to the local cellular components within the microenvironment, which largely eliminates micromotion and tissue damage associated with signal instability ${ }^{48}$. In addition, NeuE is a substrate for the migration of newborn neurons, suggesting its potential to direct cells towards sites of damage where they could help repair or regenerate damaged tissues, and as a multimodal platform for actively remodeling tissue while simultaneously 
monitoring such changes. Nevertheless, multifunctional integration that allows for simultaneous optical, electrical and chemical interrogation ${ }^{19,20}$ and high spatial integration that allows for simultaneous recording of a larger number of neurons ${ }^{26}$ represent interesting directions to pursue in the future, for example, by integrating higher resolution fabrication methods. In addition, given that the current NeuE design has ca. eliminated structural and mechanical distinctions with the fundamental neuron component of the brain, we suggest that this can serve as an ideal starting point to exploit biochemical modification strategies ${ }^{12,13}$ to endow it with added functionality. More generally, we believe that the neural interface of bioinspired NeuE, which is structurally and mechanically indistinguishable from neurons, gliosis-free, functionally stable and pro-regenerative, can open opportunities for next-generation brain-machine interfaces and biomedical therapeutics.

\section{Methods}

\section{Design and fabrication of NeuE.}

The design and structural parameters of NeuE are distinct from previous reports ${ }^{16-18}$, although the overall fabrication approach is similar to our previous reports ${ }^{16-18}$. The parameters of the two NeuE designs used for these studies are summarized in Supplementary Table 1. Barcodes based on a unique combination of triangles and circles associated with electrodes (Supplementary Fig. 11b) were also introduced for indexing the recording electrodes in electrophysiological experiments. Here we focus on 16-channel NeuE probes, but note that higher channel numbers can be achieved ${ }^{25}$ without varying the reported designs by using higher resolution fabrication ${ }^{26}$.

The key fabrication steps for NeuE probes are as follows: (1) A 100-nm thick Ni sacrificial layer was thermally evaporated (Sharon Vacuum Co.) onto a 3-inch Si wafer (n-type $0.005 \Omega$ $\mathrm{cm}, 600-\mathrm{nm}$ thermal oxide, NOVA Electronic Materials), which was pre-cleaned with oxygen plasma (100 W, 5 min; AST Products). (2) Negative photoresist SU-8 (SU-8 2000.5; MicroChem) was mixed with Lissamine ${ }^{\mathrm{TM}}$ rhodamine B ethylenediamine (RhBen; ca. 10 $\mu \mathrm{g} / \mathrm{ml}$; Thermo Fisher Scientific) and placed in the dark at room temperature (RT) for 3 days. The primary amine groups of RhBen covalently react with the epoxide groups of SU-8 to afford stable fluorescence labeling ${ }^{49}$. To eliminate any possible undissolved RhBen, the SU-8/RhBen solution was then centrifuged (Thermo Fisher Scientific) at 10,000 rpm for 3 min immediately before use. The supernatant of SU-8/RhBen solution was spin coated on the $\mathrm{Si}$ wafer to a thickness of $\sim 420 \mathrm{~nm}$, pre-baked sequentially at $65^{\circ} \mathrm{C}$ for 1 min and $95{ }^{\circ} \mathrm{C}$ for $4 \mathrm{~min}$, and then patterned by photolithography with a mask aligner (SUSS MA6 mask aligner, SUSS MicroTec). After photolithography exposure, the Si wafer was post-baked sequentially at $65^{\circ} \mathrm{C}$ for $3 \mathrm{~min}$ and $95^{\circ} \mathrm{C}$ for $3 \mathrm{~min}$. (3) The SU-8 photoresist was developed (SU-8 Developer, MicroChem) for 2 min, rinsed with isopropanol, dried with N2 and hard baked at $180^{\circ} \mathrm{C}$ for $1 \mathrm{~h}$. (4) The Si wafer was then treated with oxygen plasma (50 W, $30 \mathrm{~s}$ ), spin coated with MCC Primer 80/20 and LOR 3A resist (MicroChem), and baked at $180{ }^{\circ} \mathrm{C}$ for 5 min. For NeuE design-1, Shipley 1805 positive photoresist (Microposit, Dow Chemical Company) was spin coated on the Si wafer and baked at $115{ }^{\circ} \mathrm{C}$ for $5 \mathrm{~min}$. The positive resist was patterned by photolithography (SUSS MA6 mask aligner) and developed 
(MFCD-26, Microposit, Dow Chemical Company) for $90 \mathrm{~s}$. For smaller NeuE design-2, diluted S1805 (1:3 (vol:vol) diluted in Thinner-P, Microchem) was spin coated on the $\mathrm{Si}$ wafer and baked at $115^{\circ} \mathrm{C}$ for $1 \mathrm{~min}$. The positive resist was patterned by photolithography with a maskless aligner (MLA150) and developed (MF-CD-26) for 30 s. (5) After a flood exposure of the whole wafer, a 3-nm thick Cr layer and a 100-nm thick Au layer were sequentially deposited by electron-beam evaporation (Denton Vacuum), followed by a liftoff step (MF-CD-26) for the Au interconnect lines. (6) Steps 4 and 5 were repeated for photolithography patterning and deposition of Pt recording electrodes (3-nm-thick $\mathrm{Cr}$ and 50-nm-thick Pt). (7) Steps 2 and 3 were repeated for photolithography patterning of the top SU-8 layer as the encapsulation and insulating layer of the metal interconnect lines. After hard baking the top SU-8 layer at $180{ }^{\circ} \mathrm{C}$ for $1 \mathrm{~h}$, the Si wafer was hard baked again at 190$195^{\circ} \mathrm{C}$ for $1 \mathrm{~h}$ to allow interdiffusion of the bottom and top SU-8 layers and yield an effectively monolithic component. (8) To release NeuE probes, the Si wafer was cleaned with oxygen plasma $(50 \mathrm{~W}, 30 \mathrm{~s})$ and then transferred to a $\mathrm{Ni}$ etchant solution comprising 40\% $\mathrm{FeCl} 3: 39 \% \mathrm{HCl}: \mathrm{H} 2 \mathrm{O}=1: 1: 20$ for 60-90 min. Released NeuE probes were rinsed with deionized (DI) water, soaked in an aqueous solution of poly-D-lysine $(1.0 \mathrm{mg} / \mathrm{ml}, \mathrm{MW}$ 70,000-150,000, Sigma-Aldrich) for $24 \mathrm{~h}$ and then transferred to $1 \times$ phosphate buffered saline (PBS) solution (HyClone, GE Healthcare Life Sciences). Immediately before implantation, NeuE probes were sterilized as described in the following.

\section{Structure characterization of NeuE.}

Zeiss Ultra Plus Field Emission SEM was used to characterize the structure of NeuE building block components. The typical accelerating voltage is $0.6 \mathrm{kV}$. False colors were used for the presentation of Fig. 1b based on the raw images shown in Supplementary Fig. 2a,b, where SU-8 was rendered red and metal was rendered yellow.

\section{Impedance characterization of NeuE.}

1. The electrical impedance of each recording electrode at $1 \mathrm{kHz}$ was measured by the Intan system (Supplementary Fig. 14a), as detailed in the section Chronic electrophysiological recording from awake and restrained mice.

2. To characterize the frequency-dependent inter-channel leakage impedance, impedance was measured on adjacent SU-8 passivated Au interconnects while immersed in $1 \times$ PBS to approximate the in vivo recording environment, using an Agilent B1500A semiconductor device parameter analyzer (Agilent Technologies) with B1520AFG multifrequency capacitance measurement unit (Agilent Technologies). Measurements were taken from $1 \mathrm{kHz}$ to $10 \mathrm{kHz}$ (most relevant to single-unit spike recording).

\section{Vertebrate animal subjects.}

Adult (6-8 weeks, 25-35 g) male B6.Cg-Tg(Thy1-YFP)HJrs/J (Thy1-YFP-H, stock number 003782, Jackson Laboratory, Bar Harbor, ME), B6.Cg-Tg(Thy1-YFP)16Jrs/J (Thy1YFP-16, stock number 003709, Jackson Laboratory), FVB/N-Tg(GFAPGFP)14Mes/J (GFAP-GFP, stock number 003257, Jackson Laboratory) and C57BL/6J mice (stock number 000664, Jackson Laboratory) were used in this study. Exclusion criteria were pre- 
established: animals with substantial acute implantation damage ( $>50 \mu \mathrm{L}$ of initial liquid injection volume) or damage of a major blood vessel were discarded from further experiments.

All procedures were approved by the Animal Care and Use Committee of Harvard University. The animal care and use programs at Harvard University meet the requirements of the Federal Law (89-544 and 91-579) and NIH regulations and are also accredited by the American Association for Accreditation of Laboratory Animal Care (AAALAC). Before surgical procedures, animals were group-housed on a $12 \mathrm{~h}: 12 \mathrm{~h}$ light:dark schedule in the Harvard University Biology Research Infrastructure (BRI) and fed with food and water ad libitum as appropriate. Animals were housed individually after surgical procedures.

\section{In vivo mouse survival surgery.}

1. Stereotaxic injection of NeuE in mouse brains.-In vivo injection of NeuE probes into live mice was performed using a controlled stereotaxic injection method as described previously ${ }^{17,27}$, and reproduced here for completeness. All metal tools in direct contact with the animal subjects were bead-sterilized (Fine Science Tools) for $1 \mathrm{~h}$ before use, and all plastic tools in direct contact with the animal subjects were sterilized with $70 \%$ ethanol and rinsed with sterile DI water and sterile $1 \times$ PBS before use. The NeuE probes were sterilized with $70 \%$ ethanol followed by rinsing in sterile DI water and sterile $1 \times$ PBS before injection, and then loaded into sterile glass capillaries with an inner diameter of 400 $\mu \mathrm{m}$ and an outer diameter of $550 \mu \mathrm{m}$ (Produstrial). Mice were anesthetized by intraperitoneal (IP) injection of a mixture of $75 \mathrm{mg} / \mathrm{kg}$ (body weight) of ketamine (Patterson Veterinary Supply) and $1 \mathrm{mg} / \mathrm{kg}$ dexdomitor (Orion). The degree of anesthesia was verified via toe pinch before surgery started. A homeothermic blanket (Harvard Apparatus) was set to $37^{\circ} \mathrm{C}$ and placed underneath the anesthetized mouse. The anesthetized mouse was placed in the stereotaxic frame (Lab Standard Stereotaxic Instrument, Stoelting Co.) equipped with two ear bars and one nose clamp. Puralube vet ointment (Dechra Pharmaceuticals) was applied on both eyes to moisturize the eye surface throughout the operation. Hair removal lotion (Nair, Church \& Dwight) was applied to the scalp for depilation and Betadine surgical scrub (Purdue Products) was applied to sterilize the depilated scalp skin. A sterile scalpel was used to make a $5 \mathrm{~mm}$ longitudinal incision in the scalp along the sagittal sinus. The scalp skin was resected to expose a $5 \mathrm{~mm} \times 5 \mathrm{~mm}$ portion of the skull.

In the surgeries for electrophysiological recording, Metabond adhesive luting cement (Parkell) was applied over the exposed cranial bone to prepare the skull surface for mounting the electronics later. A 1-mm burr hole was drilled using a dental drill (Micromotor with On/Off Pedal 110/220, Grobet USA) with the stereotaxic coordinates of anteroposterior: $-4.96 \mathrm{~mm}$, mediolateral: $3.10 \mathrm{~mm}$. The dura was carefully incised and resected using a sterile 27-gauge needle (PrecisionGlide, Becton Dickinson). Then a sterilized 0-80 set screw (18-8 stainless steel cup point set screw, outer diameter $1.52 \mathrm{~mm}$, groove diameter: 1.14 $\mathrm{mm}$, length: $4.76 \mathrm{~mm}$; McMaster-Carr Supply Company) was screwed into this 1-mm burr hole to a depth of $500 \mu \mathrm{m}$ to serve as the grounding and reference electrode. Metabond adhesive luting cement was used to fix the junction between the screw and the skull. 
A 1-mm diameter burr hole was drilled for the injection of NeuE according to the following stereotaxic coordinate: anteroposterior, $-2.30 \mathrm{~mm}$; mediolateral, $1.75 \mathrm{~mm}$; dorsoventral, $2.00 \mathrm{~mm}$. The dura was removed from the burr hole, and sterile $1 \times$ PBS was swabbed on the skull surface to keep it moist throughout the surgery. NeuE was injected into the targeted brain region using the controlled injection method reported previously ${ }^{27}$. In brief, the glass needle loaded with sterilized NeuE was inserted in the micropipette holder, which was connected to a $5 \mathrm{~mL}$ syringe (Becton Dickinson), which was pre-filled with $1 \times$ PBS and mounted on a syringe pump (PHD 2000, Harvard Apparatus). The micropipette holder was mounted on a stereotaxic stage equipped with a motorized linear translation stage (860A motorizer and 460A linear stage, Newport) that could move the stereotaxic arm in the $\mathrm{z}$ direction with a constant preset velocity ranging from 0.05 to $0.5 \mathrm{~mm} / \mathrm{s}$. The needle was positioned at the surface of exposed brain tissue and then inserted into the brain tissue to the desired coordinates which, when coupled with rational design of electrode distribution, allows for the placement of electrodes at particular locations within the brain. Controllable injection was carried out by synchronizing the syringe pump with the motorized linear translation stage, with a typical liquid injection rate of $5-15 \mathrm{~mL} / \mathrm{h}$ and a typical translational stage retraction velocity of $0.2-0.5 \mathrm{~mm} / \mathrm{s}$. The volumetric flow rate and the needle retraction velocity were adjusted such that the upper part of NeuE, which was visualized through an eyepiece camera (DCC1240C, Thorlabs), remained stationary in the field of view. The total injection volume was usually 5-50 $\mu \mathrm{L}$ over $2 \mathrm{~mm}$ length of injection. Control experiments with sham injections were performed in a similar manner without NeuE implantation, but with the injection of about $30 \mu \mathrm{L}$ of $1 \times$ PBS. Additional details of the implantation process are available at http://meshelectronics.org/protocols.html\#injection.

2. Suture closure for 3D mapping surgeries.-After implantation of the NeuE, the scalp was closed with 3M Vetbond tissue adhesive. Triple antibiotic ointment (WATER-JEL Technologies) was applied copiously on the closure.

3. Electrical connection of NeuE for chronic recordings.-After injection of the NeuE into the targeted region of a mouse brain, the glass capillary was repositioned over a flexible flat cable (FFC, Premo-Flex, Molex), and then the remaining part of the NeuE was expelled from the capillary and unfolded onto the FFC to expose the input-output connection pads. Bonding of NeuE input-output pads to the FFC was carried out by a previously reported conductive ink printing method ${ }^{27}$. In brief, a carbon nanotube solution (Stock No.: P093099-11, Tubes@ Rice) was loaded into pulled glass capillary printer head with an I.D. of ca. $150 \mu \mathrm{m}$ by capillary forces. The printer head was fixed to a holder (Warner Instruments) and then mounted onto a motorized micromanipulator (MP-285/M, Sutter Instrument), controlled by a rotary optical encoder (ROE-200, Sutter Instrument, Novato, CA) and controller (MPC-200, Sutter Instrument). A custom-written LabVIEW program (available at http://meshelectronics.org/static/cnt-labview.zip) was used to set the starting and ending positions for each channel and drove the printer head to print the conductive ink in between automatically. Each channel of the NeuE could be individually connected to the corresponding channel on the FFC. Then the entire FFC with carbon nanotube-bonded NeuE was cemented to the mouse skull by applying more Metabond quick adhesive luting cement on the top and side of the FFC and the exposed mouse skull while 
the other end of the FFC was uncovered and is connected to the recording system via a custom printed circuit board (PCB) interface board. Recently, a simplified plug-and-play input-output connection methodology has been reported ${ }^{50,51}$. This strategy could be adapted to the NeuE design without affecting the crucial bioinspired implanted region of the NeuE design.

4. Postoperative care.-After surgery, each mouse was returned to a cage equipped with a $37^{\circ} \mathrm{C}$ heating pad. The activity of the mouse was monitored every hour until it was fully recovered from anesthesia. Buprenex (Buprenorphine, Patterson Veterinary Supply) analgesia was given intraperitoneally at a dose of $0.05 \mathrm{mg}$ per $\mathrm{kg}$ body weight every $12 \mathrm{~h}$ for up to $72 \mathrm{~h}$ post-surgery.

\section{D mapping of the NeuE/brain interfaces.}

1. Histology sample preparation.-Mice with implanted NeuE were anesthetized with ketamine/dexdomitor and transcardially perfused with ice-cold $40 \mathrm{ml} 1 \times \mathrm{PBS}$ and 40 $\mathrm{ml} 4 \%$ formaldehyde (Electron Microscopy Sciences) at specified times post-injection, followed by decapitation. The scalp skin was removed, and the exposed skull was ground for 10-20 min at 10,000 rpm using a high-speed rotary tool (Dremel). Brains with the NeuE undisturbed were removed from the cranium and placed in $4 \%$ formaldehyde for $24 \mathrm{~h}$ and then transferred to $1 \times$ PBS for another $24 \mathrm{~h}$ to remove any remaining formaldehyde. Brains were then embedded in 3\% agarose (SeaPlaque agarose, Lonza) hydrogel, cut into blocks of $2 \mathrm{~cm}$ (length) $\times 2 \mathrm{~cm}$ (width) $\times 1 \mathrm{~cm}$ (height). Another NeuE probe was injected into the agarose hydrogel block to serve as a reference for the measurement of distance in subsequent micro-computed tomography (micro-CT) and vibratome sectioning steps (Supplementary Fig. 3). The hydrogel block containing the mouse brain was imaged by a micro-CT X-ray scanning system (HMX ST 225, Nikon Metrology) controlled by Inspect-X software (Nikon Metrology). CT Pro 3D software (Nikon Metrology) was used to calibrate centers of rotation and to reconstruct the images. VGStudio MAX 3.0 software (Volume Graphics) was used to render and analyze the 3D reconstructed images. Distances between the reference NeuE and target NeuE as well as the angle $\theta$ (usually less than $5^{\circ}$ ) between the longitudinal axis of the target $\mathrm{NeuE}$ and the reference surface were measured from reconstructed images (Supplementary Fig. 3). The reference surface of the hydrogel block was then cut using a homemade sectioning stage according to measured angle $\theta$ to make this surface parallel to the longitudinal axis of the target NeuE. Finally, a mouse brain was sectioned into 500-1000 $\mu \mathrm{m}$ slices using a vibratome (VT1000 S vibrating blade microtome, Leica). The reference NeuE was used as a landmark to indicate the distance to the target NeuE to facilitate accurate sectioning. Because fluorescence from the target NeuE can be visualized with the aid of a wide-field epifluorescence microscope (Olympus) when it is 50$100 \mu \mathrm{m}$ near the tissue surface, it is possible to adjust the sectioning such that one tissue slice includes the entire NeuE probe.

2. Tissue clearing and immunostaining.-Transgenic mouse samples with endogenous expression of fluorescent proteins were used directly for refractive index matching and microscopy imaging as described below in the next two sections. Mouse brain slices that were targeted for immunostaining were first cleared using procedures adapted 
from the tissue clearing techniques CLARITY ${ }^{30}$ and $\mathrm{PACT}^{31}$. After vibratome sectioning, brain slices were placed in $1 \times$ PBS containing $4 \%(\mathrm{w} / \mathrm{v})$ acrylamide (Sigma-Aldrich) and $0.25 \%(\mathrm{w} / \mathrm{v})$ VA-044 thermal polymerization initiator (Fisher Scientific) at $4{ }^{\circ} \mathrm{C}$ for 3 days. The solution was replaced with fresh solution immediately before placing the brain slices in X-CLARITYTM polymerization system (Logos Biosystems) for $3 \mathrm{~h}$ at $37^{\circ} \mathrm{C}$. After polymerization, any remaining gel from the tissue surface was removed and the slices were rinsed with PBST ( $1 \times$ PBS with $0.2 \%$ Triton X-100, Thermo Fisher Scientific) before placing in electrophoretic tissue clearing solution (Logos Biosystems) at $37{ }^{\circ} \mathrm{C}$ for $3-5$ days until the samples were translucent. After clearing, the brain slices were rinsed once with PBST containing 0.3 M glycine (Sigma-Aldrich), placed in fresh PBST containing 0.3 M glycine and incubated at $4{ }^{\circ} \mathrm{C}$ overnight. The samples were then placed in fresh PBST and gently shaken for $5 \mathrm{~h}$, with the PBST replaced by fresh solution every hour. The brain slices were incubated with 1:100-1:200 primary antibodies, rabbit anti-doublecortin (Abcam) and/or rat anti-GFAP (Thermo Fisher Scientific) and/or goat anti-Iba1 (Abcam) in PBST for 4 days at $4{ }^{\circ} \mathrm{C}$. After incubation, slices were placed in fresh PBST at RT to let excess antibody diffuse out of the tissue. The PBST was replaced with fresh solution every $8 \mathrm{~h}$ over the course of 2 days. Then the samples were incubated with 1:100-1:200 secondary antibodies, goat anti-rabbit Alexa Fluor 488 (Abcam) or goat anti-rabbit Alexa Fluor 647 (Abcam), or donkey anti-rabbit Alexa Fluor 488 (Abcam) and/or donkey anti-rat Alexa Fluor 647 (Abcam) and/or donkey anti-goat Alexa Fluor 594 (Abcam) in PBST for 4 days at $4{ }^{\circ} \mathrm{C}$. Slices were placed in fresh PBST containing $1 \mu \mathrm{g} / \mathrm{ml}$ DAPI (Sigma-Aldrich) at RT to let excess antibody diffuse out of the tissue and simultaneously stain nuclear DNA. The PBST with DAPI was replaced with fresh solution every $8 \mathrm{~h}$ over the course of 2 days. Note that DAPI was initially dissolved in DI water then diluted with PBST to make a concentrated stock solution $(25 \mu \mathrm{g} / \mathrm{ml})$ that was stored at $4{ }^{\circ} \mathrm{C}$ for no longer than one month before making a fresh stock. If necessary to improve light penetration for microscopy imaging, slices could be further incubated in Tris (Sigma-Aldrich) with 2\% SDS (Sigma-Aldrich) and $0.3 \mathrm{M}$ glycine at $\mathrm{pH} 6.8$ at RT for 3 days then re-stained with DAPI.

3. Refractive index (RI) matching.-Brain slices were glued at their edge to the bottom of 50-mm-diameter petri dishes by Devcon 5 Minute Epoxy (ITW Polymers Adhesives) and then incubated in RI matching solution PROTOS ${ }^{52}$ (diatrizoic acid, SigmaAldrich; N-Methyl-D-glucamine, Sigma-Aldrich; OptiPrep, Accurate Chemical and Scientific) or $80 \%$ glycerol:20\% PBS (glycerol, Sigma-Aldrich) $24 \mathrm{~h}$ prior to microscopy imaging.

4. Microscopy imaging.-Fluorescence images were acquired on a Zeiss LSM 880 confocal/multiphoton microscope (Carl Zeiss Microscopy) with a 20x objective (numerical aperture $=1.0$, free working distance $=5.6 \mathrm{~mm}$ ). Near infrared lasers were used for twophoton excitation of endogenously expressed fluorescent proteins in the transgenic mouse brain slices. Specifically, an $840 \mathrm{~nm}$ laser was used to excite $\mathrm{RhBen}$, a $950 \mathrm{~nm}$ laser to excite YFP and $920 \mathrm{~nm}$ laser to excite GFP. The bandpass filter for RhBen was 575-610 nm and that for YFP and GFP was 500-550 nm. Confocal images of cleared and immunostained brain slices were acquired using 405, 488, 561 and/or $633 \mathrm{~nm}$ lasers as the excitation sources for DAPI, Alexa Fluor 488, RhBen/Alexa Fluor 594 and/or Alexa Fluor 647, respectively. 
The corresponding bandpass filters were 411-496, 500-553, 562-624, 624-660 and/or 677$755 \mathrm{~nm}$, respectively. Images were acquired by taking a tile scan together with $\mathrm{Z}$ stacks, with a voxel size of $0.2-0.6 \mu \mathrm{m}(\mathrm{x}) \times 0.2-0.6 \mu \mathrm{m}(\mathrm{y}) \times 0.4-2.5 \mu \mathrm{m}(\mathrm{z})$ and tile scan overlap of $10 \%$. YFP, GFP (GFAP), Iba-1, DCX, DAPI and NeuE are represented as green, cyan, magenta, yellow, blue and red in the composite images.

\section{Chronic electrophysiological recording from awake and restrained mice.}

The following procedures were performed according to previous works ${ }^{17}$ and are reproduced here for completeness. The electrophysiology of mice with implanted NeuE was recorded every day for $\sim 30$ min during the first half week post-implantation, every other day until the second week, followed by twice a week thereafter. Mice were restrained in a Tailveiner restrainer (Braintree Scientific) while their head-mounted FFCs were connected to an Intan RHD 2132 amplifier evaluation system (Intan Technologies) through a homemade printed circuit board. 0-80 set screws were used as references. Electrophysiological recordings were acquired with a $20-\mathrm{kHz}$ sampling rate and a $60-\mathrm{Hz}$ notch filter, while the electrical impedance of each recording electrode at $1 \mathrm{kHz}$ was also measured by the same system and showed consistent values of $<1 \mathrm{M} \Omega$ that allow for single unit recording measurements (Supplementary Fig. 14a). Thy 1-YFP-16 mice were usually recorded for 3 months until they were transcardially perfused and underwent the same histology sample preparation procedure as described in the above section '3D mapping of the NeuE/brain interfaces'.

\section{Supplementary Material}

Refer to Web version on PubMed Central for supplementary material.

\section{Acknowledgements}

The authors thank D. Richardson and S. Terclavers for help with image acquisition, data handling, and critical discussion, and J. Huang for assistance with recording instrumentation. This work is supported by National Institute on Drug Abuse of the National Institutes of Health (1R21DA043985-01), a NIH Director's Pioneer Award (1DP1EB025835-01) and the Air Force Office of Scientific Research (FA9550-14-1-0136) (to C.M.L.), the Simmons Awards (to X.Y.), and the American Heart Association Postdoctoral Fellowship (16POST27250219) and the NIH Pathway to Independence Award (1K99AG056636-02) (to G.H.). This work was performed in part at the Harvard Center for Biological Imaging (HCBI), and Harvard University Center for Nanoscale Systems (CNS), a member of the National Nanotechnology Coordinated Infrastructure Network (NNCI) supported by the National Science Foundation.

\section{References}

1. Ma X et al. Tuning crystallization pathways through sequence engineering of biomimetic polymers. Nat. Mater 16, 767-774 (2017). [PubMed: 28414316]

2. Fratzl P, Kolednik O, Fischer FD \& Dean MN The mechanics of tessellations - bioinspired strategies for fracture resistance. Chem. Soc. Rev 45, 252-267 (2016). [PubMed: 26377507]

3. Green JJ \& Elisseeff JH Mimicking biological functionality with polymers for biomedical applications. Nature 540, 386-394 (2016). [PubMed: 27974772]

4. Sadtler K et al. Design, clinical translation and immunological response of biomaterials in regenerative medicine. Nat. Rev. Mater 1, 16040 (2016).

5. Chen R, Canales A \& Anikeeva P Neural recording and modulation technologies. Nat. Rev. Mater 2, 16093 (2017). 
6. Feiner R \& Dvir T Tissue-electronics interfaces: from implantable devices to engineered tissues. Nat. Rev. Mater 3, 17076 (2017).

7. Shoffstall AJ \& Capadona JR Bioinspired materials and systems for neural interfacing. Curr. Opin. Biomed. Eng 6, 110-119 (2018).

8. Capadona JR, Shanmuganathan K, Tyler DJ, Rowan SJ \& Weder C Stimuli-Responsive Polymer Nanocomposites Inspired by the Sea Cucumber Dermis. Science 319, 1370-1374 (2008). [PubMed: 18323449]

9. Smith DW et al. Internal Jugular Vein Compression Mitigates Traumatic Axonal Injury in a Rat Model by Reducing the Intracranial Slosh Effect. Neurosurgery 70, 740-746 (2012). [PubMed: 21904255]

10. Polikov VS, Tresco PA \& Reichert WM Response of brain tissue to chronically implanted neural electrodes. J. Neurosci. Methods 148, 1-18 (2005). [PubMed: 16198003]

11. Salatino JW, Ludwig KA, Kozai TDY \& Purcell EK Glial responses to implanted electrodes in the brain. Nat. Biomed. Eng 1, 862-877 (2017). [PubMed: 30505625]

12. Kozai TDY et al. Ultrasmall implantable composite microelectrodes with bioactive surfaces for chronic neural interfaces. Nat. Mater 11, 1065-1073 (2012). [PubMed: 23142839]

13. Charkhkar $\mathrm{H}$ et al. Chronic intracortical neural recordings using microelectrode arrays coated with PEDOT-TFB. Acta Biomater. 32, 57-67 (2016). [PubMed: 26689462]

14. Bedell HW et al. Targeting CD14 on blood derived cells improves intracortical microelectrode performance. Biomaterials 163, 163-173 (2018). [PubMed: 29471127]

15. Arati S, Subramaniam DR \& Jit M Long-term changes in the material properties of brain tissue at the implant-tissue interface. J. Neural Eng. 10, 066001 (2013). [PubMed: 24099854]

16. Liu J et al. Syringe-injectable electronics. Nat. Nanotechnol 10, 629-636 (2015). [PubMed: 26053995]

17. Fu T-M et al. Stable long-term chronic brain mapping at the single-neuron level. Nat. Methods 13, 875-882 (2016). [PubMed: 27571550]

18. Zhou T et al. Syringe-injectable mesh electronics integrate seamlessly with minimal chronic immune response in the brain. Proc. Natl. Acad. Sci. U.S.A 114, 5894-5899 (2017). [PubMed: 28533392]

19. Canales A et al. Multifunctional fibers for simultaneous optical, electrical and chemical interrogation of neural circuits in vivo. Nat. Biotechnol 33, 277-284 (2015). [PubMed: 25599177]

20. Park S et al. One-step optogenetics with multifunctional flexible polymer fibers. Nat. Neurosci 20 , 612-619 (2017). [PubMed: 28218915]

21. Minev IR et al. Electronic dura mater for long-term multimodal neural interfaces. Science 347, 159-163 (2015). [PubMed: 25574019]

22. Luan L et al. Ultraflexible nanoelectronic probes form reliable, glial scar-free neural integration. Sci. Adv 3, e1601966 (2017). [PubMed: 28246640]

23. Garcia J, Pena J, McHugh S \& Jerusalem A A Model of the Spatially Dependent Mechanical Properties of the Axon During Its Growth. Comput. Model. Eng. Sci 87, 411-432 (2012).

24. Wang SS-H et al. Functional Trade-Offs in White Matter Axonal Scaling. J. Neurosci 28, 40474056 (2008). [PubMed: 18400904]

25. Fu T-M, Hong G, Viveros RD, Zhou T \& Lieber CM Highly scalable multichannel mesh electronics for stable chronic brain electrophysiology. Proc. Natl. Acad. Sci. U.S.A 114, E10046E10055 (2017). [PubMed: 29109247]

26. Jun JJ et al. Fully integrated silicon probes for high-density recording of neural activity. Nature 551, 232-236 (2017). [PubMed: 29120427]

27. Hong GS et al. Syringe Injectable Electronics: Precise Targeted Delivery with Quantitative Input/ Output Connectivity. Nano Lett. 15, 6979-6984 (2015). [PubMed: 26317328]

28. Feng G et al. Imaging Neuronal Subsets in Transgenic Mice Expressing Multiple Spectral Variants of GFP. Neuron 28, 41-51 (2000). [PubMed: 11086982]

29. Zhuo L et al. Live Astrocytes Visualized by Green Fluorescent Protein in Transgenic Mice. Dev. Biol 187, 36-42 (1997). [PubMed: 9224672] 
30. Chung K et al. Structural and molecular interrogation of intact biological systems. Nature 497, 332-337 (2013). [PubMed: 23575631]

31. Yang B et al. Single-Cell Phenotyping within Transparent Intact Tissue through Whole-Body Clearing. Cell 158, 945-958 (2014). [PubMed: 25088144]

32. Saxena T \& Bellamkonda RV A sensor web for neurons. Nat. Mater 14, 1190-1191 (2015). [PubMed: 26585085]

33. Igarashi KM, Lu L, Colgin LL, Moser M-B \& Moser EI Coordination of entorhinal-hippocampal ensemble activity during associative learning. Nature 510, 143-147 (2014). [PubMed: 24739966]

34. Jackson A \& Fetz EE Compact Movable Microwire Array for Long-Term Chronic Unit Recording in Cerebral Cortex of Primates. J. Neurophysiol 98, 3109-3118 (2007). [PubMed: 17855584]

35. Dickey AS, Suminski A, Amit Y \& Hatsopoulos NG Single-Unit Stability Using Chronically Implanted Multielectrode Arrays. J. Neurophysiol 102, 1331-1339 (2009). [PubMed: 19535480]

36. Quiroga RQ, Nadasdy Z \& Ben-Shaul Y Unsupervised Spike Detection and Sorting with Wavelets and Superparamagnetic Clustering. Neural Comput. 16, 1661-1687 (2004). [PubMed: 15228749]

37. Schmitzer-Torbert N, Jackson J, Henze D, Harris K \& Redish AD Quantitative measures of cluster quality for use in extracellular recordings. Neuroscience 131, 1-11 (2005). [PubMed: 15680687]

38. Schmitzer-Torbert N \& Redish AD Neuronal Activity in the Rodent Dorsal Striatum in Sequential Navigation: Separation of Spatial and Reward Responses on the Multiple T Task. J. Neurophysiol 91, 2259-2272 (2004). [PubMed: 14736863]

39. Spratley JPF, Ward MCL \& Hall PS Bending characteristics of SU-8. IET Micro \& Nano Letters 2, 20-23 (2007).

40. Jog MS et al. Tetrode technology: advances in implantable hardware, neuroimaging, and data analysis techniques. J. Neurosci. Methods 117, 141-152 (2002). [PubMed: 12100979]

41. Buzsáki G et al. Tools for Probing Local Circuits: High-Density Silicon Probes Combined with Optogenetics. Neuron 86, 92-105 (2015). [PubMed: 25856489]

42. Weiler $\mathrm{S}$ et al. High-yield in vitro recordings from neurons functionally characterized in vivo. Nat. Protoc 13, 1275-1293 (2018). [PubMed: 29748648]

43. Cossell $\mathrm{L}$ et al. Functional organization of excitatory synaptic strength in primary visual cortex. Nature 518, 399-403 (2015). [PubMed: 25652823]

44. Liu X et al. Optogenetic stimulation of a hippocampal engram activates fear memory recall. Nature 484, 381-385 (2012). [PubMed: 22441246]

45. Gonçalves JT, Schafer ST \& Gage FH Adult Neurogenesis in the Hippocampus: From Stem Cells to Behavior. Cell 167, 897-914 (2016). [PubMed: 27814520]

46. Ming G. 1. \& Song H Adult Neurogenesis in the Mammalian Central Nervous System. Annu. Rev. Neurosci 28, 223-250 (2005). [PubMed: 16022595]

47. Reza M et al. In vivo migration of endogenous brain progenitor cells guided by an injectable peptide amphiphile biomaterial. J. Tissue Eng. Regen. Med 12, e2123-e2133 (2018). [PubMed: 29327429]

48. James CB et al. Failure mode analysis of silicon-based intracortical microelectrode arrays in nonhuman primates. J. Neural Eng 10, 066014 (2013). [PubMed: 24216311]

49. Rozenberg B Kinetics, Thermodynamics and Mechanism of Reactions of Epoxy Oligomers with Amines. Adv. Polym. Sci 75, 113-165 (1986).

50. Schuhmann TG, Yao J, Hong G, Fu T-M \& Lieber CM Syringe-Injectable Electronics with a Plugand-Play Input/Output Interface. Nano Lett. 17, 5836-5842 (2017). [PubMed: 28787578]

51. Schuhmann TG et al. Syringe-injectable Mesh Electronics for Stable Chronic Rodent Electrophysiology. J. Vis. Exp 137, e58003 (2018).

52. Murray E et al. Simple, Scalable Proteomic Imaging for High-Dimensional Profiling of Intact Systems. Cell 163, 1500-1514 (2015). [PubMed: 26638076] 
a
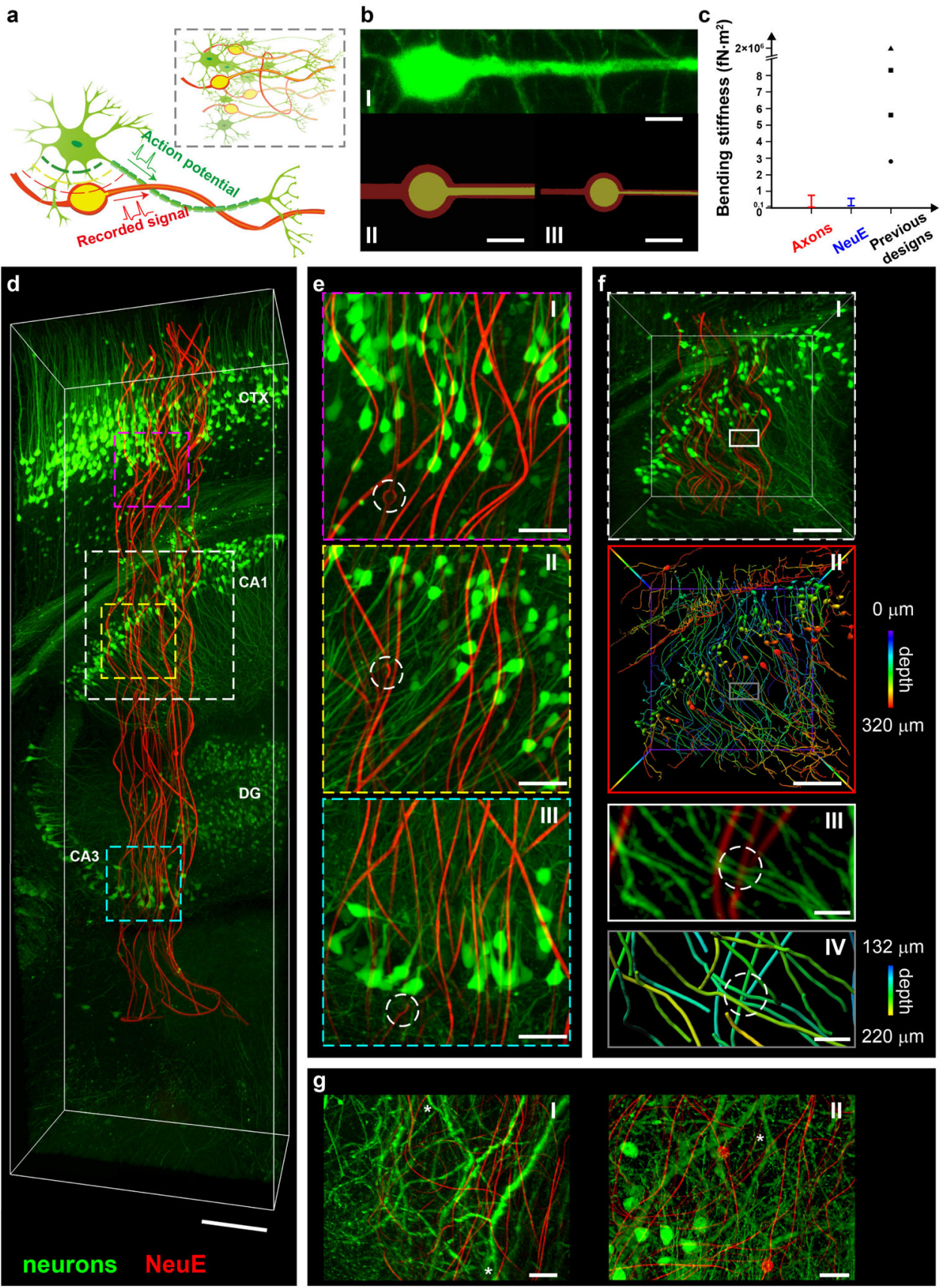

1)

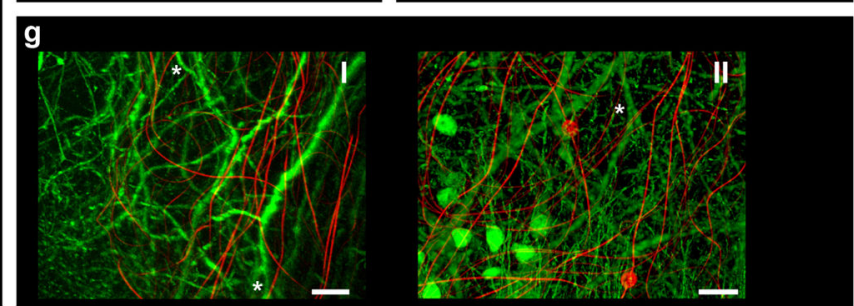

Fig. 1 . Design and characterization of NeuE, and 3D mapping of its neural interface.

a, Schematics showing the structural similarity between NeuE and neurons from the subcellular level to the network level (inset). Neurons, green; electrodes and interconnects, yellow; polymer layers, red. b. Fluorescence microscope image of a neuron (I) and falsecolored scanning electron microscope (SEM) images of two NeuE designs (II and III). Raw SEM images are shown in Supplementary Fig. 2. Scale bars, $10 \mu \mathrm{m}$. c, Bending stiffness of axons, NeuE and examples of previously reported state-of-the-art mesh ${ }^{17}$ (circle), fiber ${ }^{19,20}$ (triangle) and thread ${ }^{22}$ probes (squares). d, 3D reconstructed interface between neurons (green) and NeuE (red) at 6 weeks post-implantation. Scale bar, $200 \mu \mathrm{m}$. 3D mapping was 
repeated on $\mathrm{N}=3$ independent samples. Additional fluorescence images and quantitative analyses are shown in Supplementary Figs. 6 and 7. e, High-resolution images of the volumes highlighted by magenta (I), yellow (II) and cyan (III) dashed boxes in d. Electrodes are indicated by white dashed circles. Scale bars, $50 \mu \mathrm{m}$. f, Close up images of the white dashed box in $\mathbf{d}$. I and II correspond to standard fluorescence and depth-coded images, respectively. III and IV are close-up views indicated by the white and gray boxes in I and II, respectively, highlighting the junction between neurites and the NeuE neurite-like interconnect (white dashed circles). Color codes for depth are shown in the frame in II and the color bars to the right. Scale bars, $100 \mu \mathrm{m}$ (I and II), $20 \mu \mathrm{m}$ (III and IV). g, Close-up 3D neural interface of the smaller NeuE (b, III) in additional independent samples near cornu ammonis 1 (CA1) (I) and dentate gyrus (DG) (II) at 2 weeks post-injection. White asterisks indicate dendritic branches. Scale bars, $20 \mu \mathrm{m}$. 

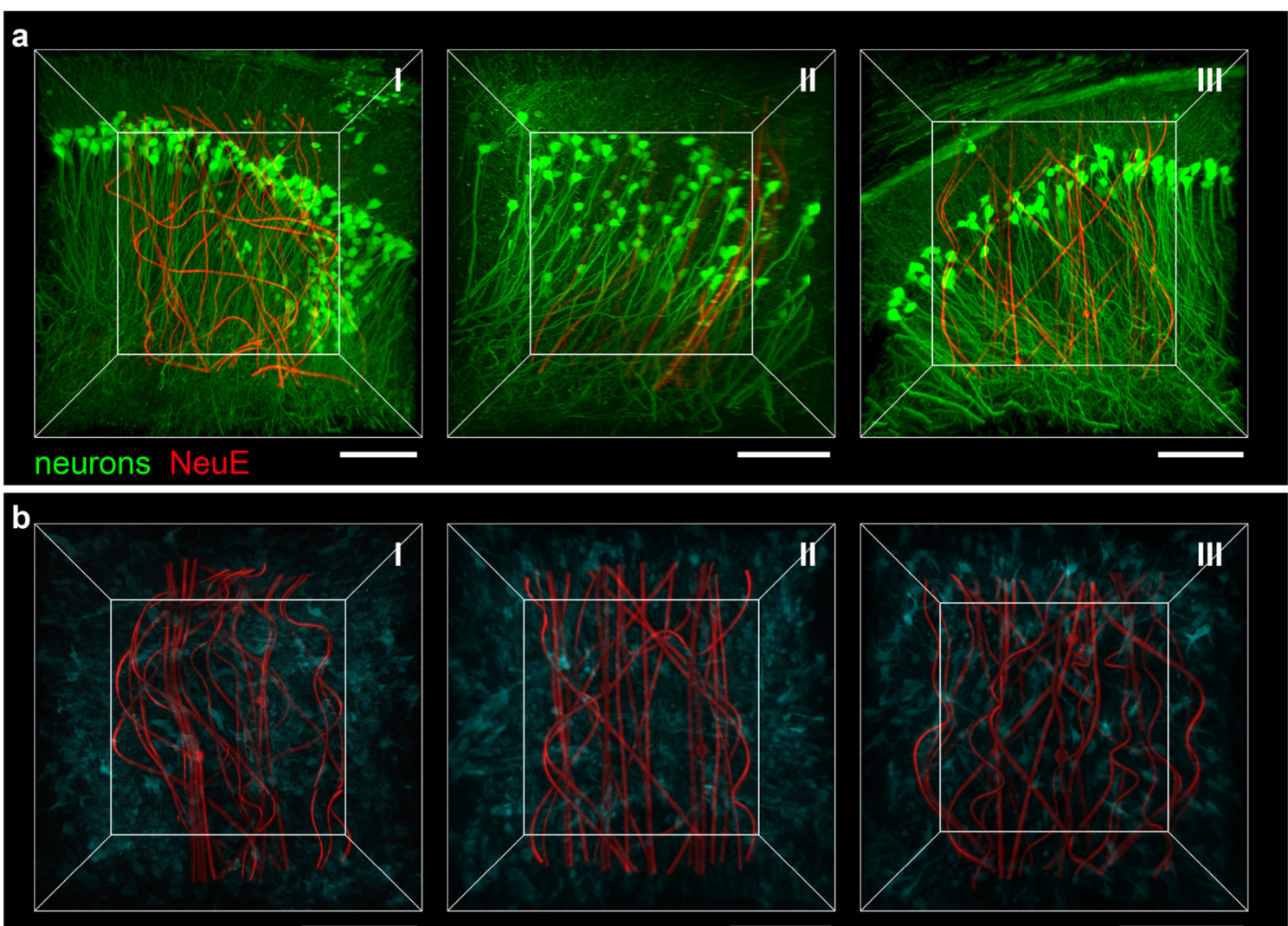

astrocytes NeuE
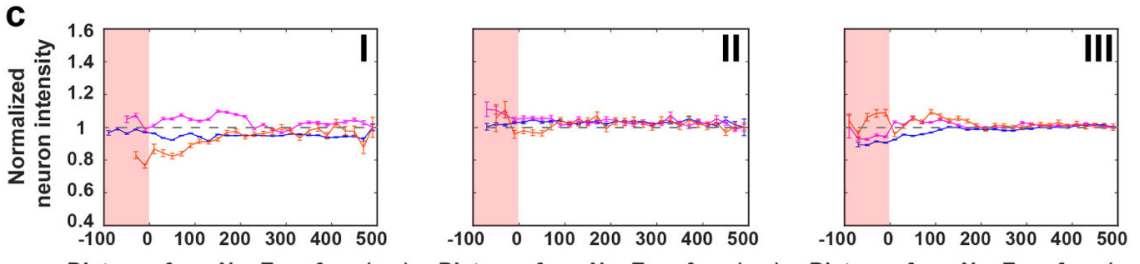

III

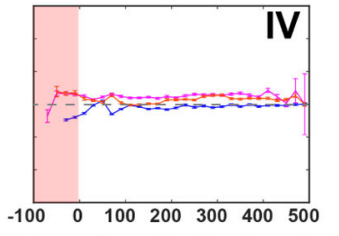

Distance from NeuE surface $(\mu \mathrm{m})$

Distance from NeuE surface $(\mu \mathrm{m})$

Distance from NeuE surface $(\mu \mathrm{m})$

Distance from NeuE surface $(\mu \mathrm{m})$

d
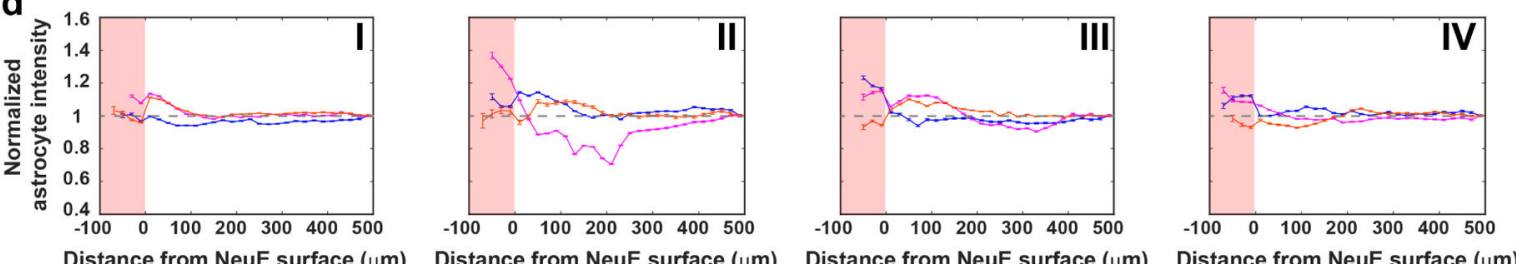

Fig. 2 | Time-dependent 3D histology studies of NeuE/brain interfaces.

a, 3D interfaces between NeuE (red) and neurons (green) at 2 days (I), 2 weeks (II) and 3 months (III) post-implantation. Scale bars, $100 \mu \mathrm{m}$. b, 3D interfaces between NeuE (red) and astrocytes (cyan) at 2 days (I), 2 weeks (II) and 3 months (III) post-implantation. Scale bars, $100 \mu \mathrm{m}$. c,d, Normalized fluorescence intensity of neurons (c) and astrocytes (d) as a function of distance from the 3D NeuE boundary at CTX (orange), hippocampal CA1 (magenta) and DG (blue) at 2 days (I), 2 weeks (II), 6 weeks (III) and 3 months (IV) postimplantation. The pink-shaded regions indicate tissue volumes within the interior of the NeuE. The relative signal was obtained by normalizing the fluorescence intensity with the 
baseline value defined as the fluorescence intensity averaged over a range of 480-500 $\mu \mathrm{m}$ away (gray dashed horizontal lines; Supplementary Note 4). The tissue volumes for analysis depend on the specific structures of the distinct brain regions as shown in Supplementary Fig. 5a,b. All error bars reflect \pm 1 s.e.m. There is substantial neuronal density, $91 \pm 12 \%$ (mean \pm s.d.) of baseline, in the interior of the NeuE probe boundary as early as 2 days, and this increases to $100 \pm 7 \%$ of baseline at times extending from 2 weeks when the neurons exhibit a fully endogenous distribution in the DG, CA1 and CTX regions. The astrocyte density is $107 \pm 8 \%$ of baseline at 2 and 14 days, and is uniform at the endogenous level, $102 \pm 3 \%$, at longer times. Time-dependent $3 \mathrm{D}$ histology studies have been repeated on $\mathrm{N}=3$ independent samples; additional fluorescence images and quantitative analyses are shown in Supplementary Figs. 6 and 7. 
a

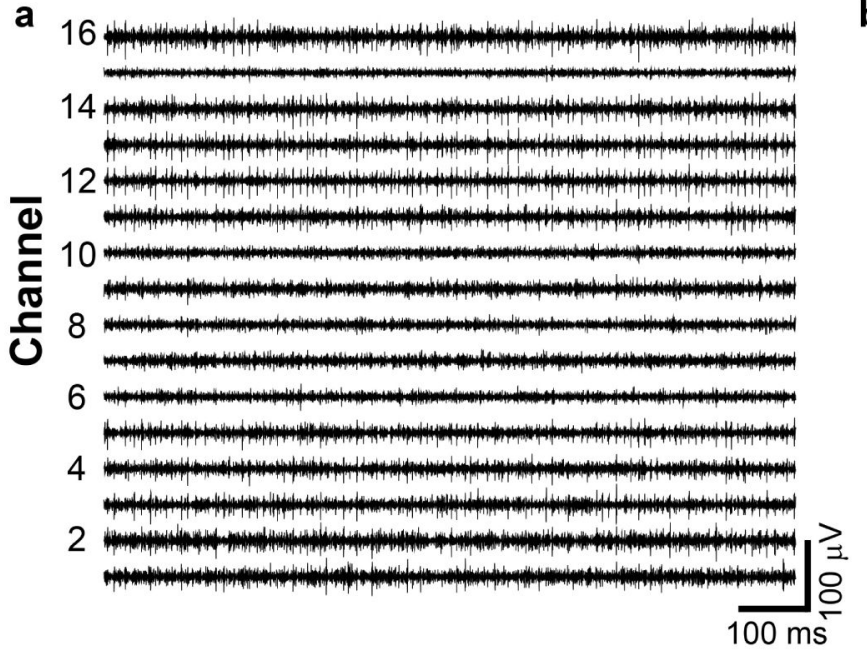

c

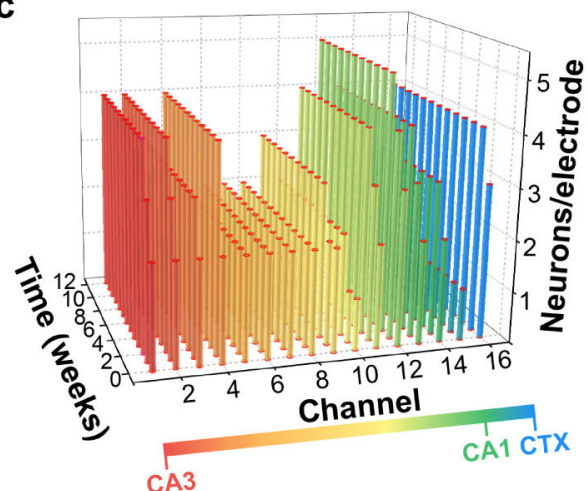

d b

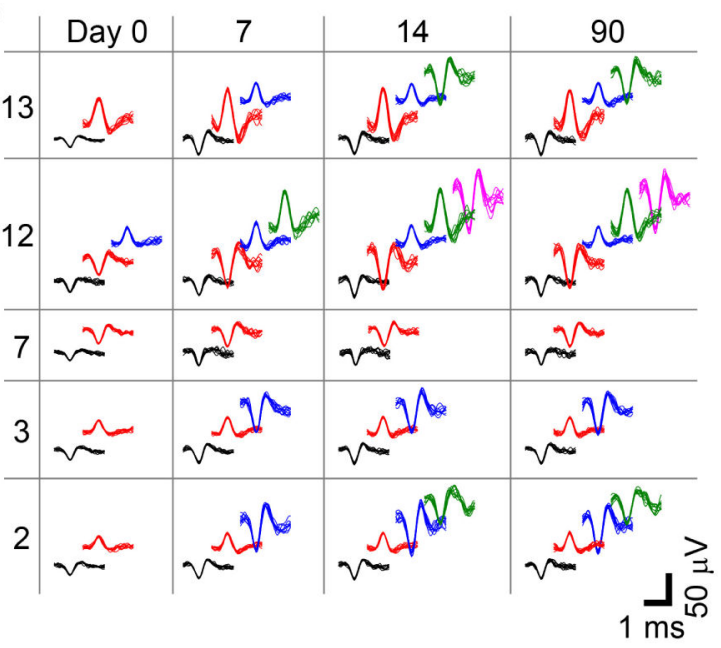

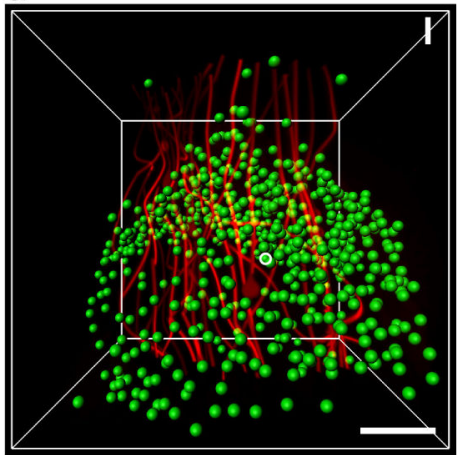

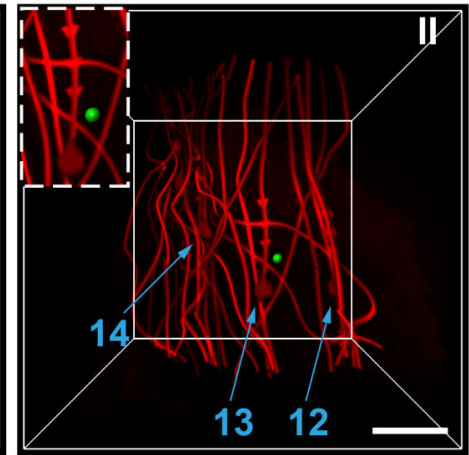

IV

Fig. 3 |. Functional interrogation with NeuE.

a, Representative 16-channel single-unit spike traces at 7 days post-injection. The $\mathrm{x}$ and $\mathrm{y}$ axes represent recording time and voltage. $\mathbf{b}$, Time evolution of spikes of principal component analysis clustered single units from five representative channels over 3 months post-injection. For each channel, each distinct color in the sorted spikes represents a unique identified neuron. c, 3D bar chart of the number of distinct neurons recorded per electrode as a function of the time post-injection. Bar colors are coded according to the brain regions as shown in the color bar at the bottom. Larger numbers of isolated neuron signals were recorded in channels located in CA3 and CA1 regions of the HIP, and lower numbers in channels located in the lower density space between CA3 and CA1. Time-dependent electrophysiology studies were repeated on $\mathrm{N}=3$ independent animals. Data and analyses from additional replicates are shown in Supplementary Figs. 18 and 19. d, 3D images of NeuE near the CA1 subfield. Green dots in I indicate the soma of imaged neurons after segmentation. The neuron triangulated by electrodes 12,13 and 14 is highlighted by a white circle. II shows the relative positioning of the triangulated neuron (green dot) with respect to electrodes 12, 13 and 14 (highlighted by light blue arrows), with an inset highlighting the triangulated neuron. Scale bars, $50 \mu \mathrm{m}$. 3D mapping and channel-indexing of all the electrodes $(\mathrm{N}=16)$ are shown in Supplementary Fig. 11. 


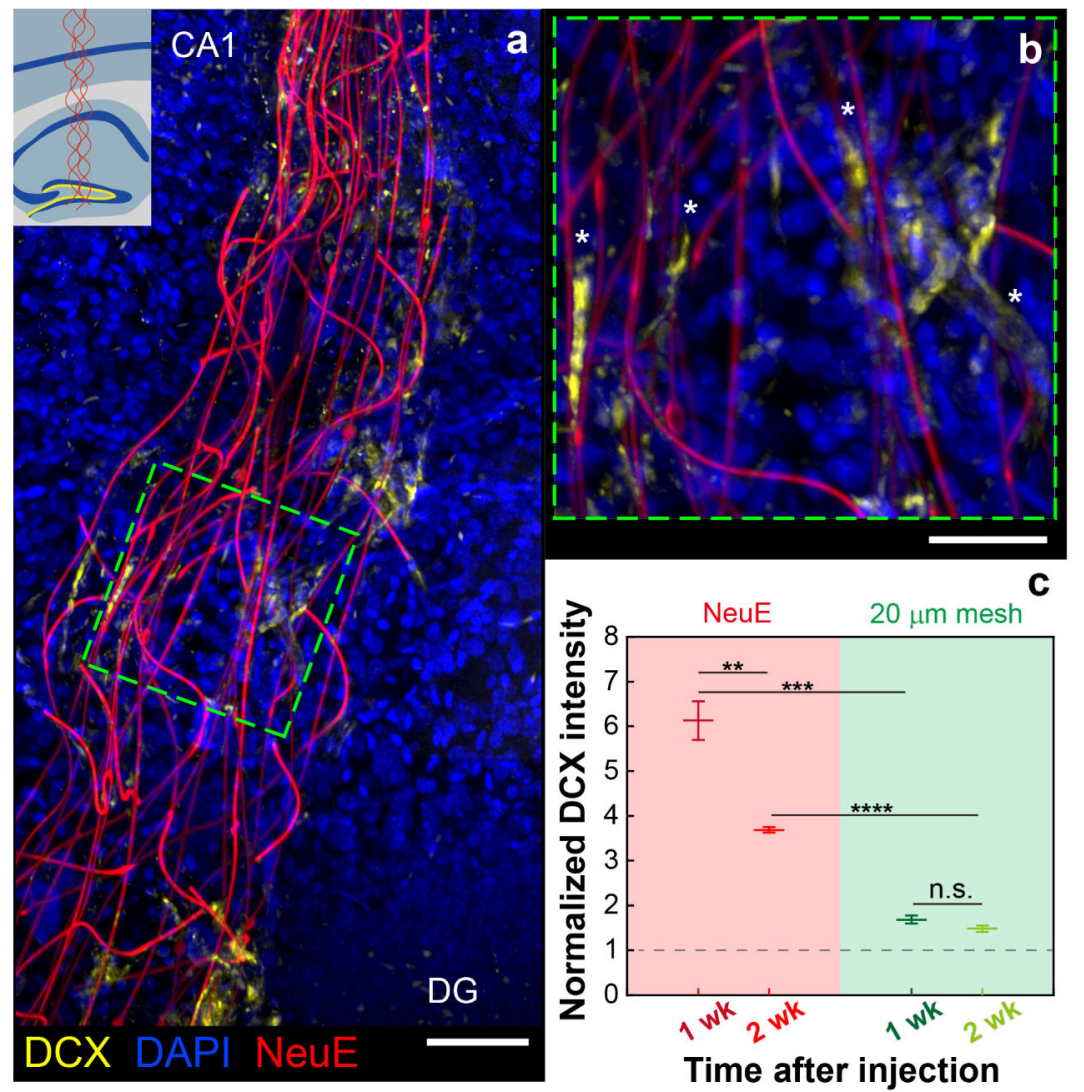

Fig. 4 |. NeuE facilitates migration of NPC-derived newborn neurons.

a, A representative $3 \mathrm{D}$ image showing the distribution of $\mathrm{DCX}^{+}$newborn neurons along $\mathrm{NeuE}$ at 1 week post-implantation. Yellow, blue and red colors represent DCX, DAPI and NeuE, respectively. Scale bar, $100 \mu \mathrm{m}$. Inset, Schematic showing the injection site, with dark blue, yellow and red colors indicating principal cell body layers, SGZ and NeuE, respectively. $\mathbf{b}$, Magnified view of the green dashed box in $\mathbf{a}$, demonstrating that some DCX + cells show neurite spreading associated and aligned with the neurite-like NeuE structure (highlighted by white asterisks). Scale bar, $50 \mu \mathrm{m}$. c, Normalized DCX intensity at 0-20 $\mu \mathrm{m}$ near the NeuE or $20 \mu \mathrm{m}$ mesh normalized against baseline values remote to the probe (gray dashed horizontal line; Supplementary Note 4). Each condition was repeated on three independent tissue volumes. Additional data are shown in Supplementary Figs. 20 and 21. All error bars reflect \pm 1 s.e.m. Top to bottom, $P=5 \times 10^{-3}\left(* * P<1 \times 10^{-2}\right), P=6 \times 10^{-4}(* * * P$ $\left.<1 \times 10^{-3}\right), P=2 \times 10^{-5}\left(* * * * P<1 \times 10^{-4}\right), P=1.4 \times 10^{-1}$ (NS, not significant, $\left.P>5 \times 10^{2}\right)$; two-tailed t-test. 